\title{
Implementation of diffusion-weighted magnetic resonance imaging in target delineation of central lung cancer accompanied with atelectasis in precision radiotherapy
}

\author{
XINLI ZHANG ${ }^{1,2}$, ZHENG FU $^{1}$, GUANZHONG GONG ${ }^{1}$, HONG WEI $^{1}$, JINGHAO DUAN $^{1}$, \\ ZHAOQIU CHEN $^{1}$, XIANGMING CHEN ${ }^{2}$, RUOZHENG WANG ${ }^{3}$ and YONG YIN ${ }^{1}$ \\ ${ }^{1}$ Department of Radiation Oncology, Shandong Cancer Hospital and Institute, Shandong University, Jinan, Shandong 250117; \\ ${ }^{2}$ Department of Medical Oncology, Tai'an City Central Hospital, Tai'an, Shandong 271000; \\ ${ }^{3}$ Department of Radiation Oncology, Affiliated Tumor Hospital of Xinjiang Medical University, \\ Urumqi, Xinjiang 830011, P.R. China
}

Received November 22, 2015; Accepted April 21, 2017

DOI: $10.3892 / 01.2017 .6479$

\begin{abstract}
Radiotherapy, particularly the target delineation of cancer based on scanned images, plays a key role in the planning of cancer treatment. Recently, diffusion-weighted magnetic resonance imaging (DW-MRI) has emerged as a prospective superior procedure compared with intensified computed tomography (CT) and positron emission tomography (PET) in the target delineation of cancer. However, the implication of DW-MRI in lung cancer, the leading cause of cancer-associated mortality worldwide, has not been extensively evaluated. In the present study, the gross target volumes of lung cancer masses delineated using the DW-MRI, CT and PET procedures were compared in a pairwise manner in a group of 27 lung cancer patients accompanied with atelectasis of various levels. The data showed that compared with CT and PET procedures, DW-MRI has a more precise delineation of lung cancer while exhibiting higher reproducibility. Together with the fact that it is non-invasive and cost-effective, these data demonstrate the great application potential of the DW-MRI procedure in cancer precision radiotherapy.
\end{abstract}

\section{Introduction}

Lung cancer is the leading cause of cancer-associated mortality worldwide (1). Radiotherapy, alone or in combination with either surgery or systematic therapies, is a key treatment modality in the curative treatment of lung cancer patients,

Correspondence to: Dr Yong Yin, Department of Radiation Oncology, Shandong Cancer Hospital and Institute, Shandong University, 440 Jiyan Road, Jinan, Shandong 250117, P.R. China E-mail: yongyinsd@163.com

Key words: diffusion-weighted magnetic resonance imaging, computed tomography, positron emission tomography, gross target volume, central lung cancer, atelectasis particularly those with advanced-stage disease (2). With the advance of radiotherapy technologies, precision radiotherapy has greatly improved the outcome for patients with lung cancer, while reducing the toxicity of an increased dose and alleviating the risk to adjacent organs. Notably, the precision delineation of the gross target volume (GTV) based on image data serves an essential role in precision radiotherapy. Several non-invasive procedures, including intensified computed tomography (CT) and positron emission tomography (PET), have been widely used for the target volume delineation of lung cancer (3). Precision radiotherapy based on CT imaging generates a risk to the organs due to its poor soft tissue contrast, particularly for central lung cancer accompanied with pulmonary atelectasis or mediastinal node metastasis (4).

Although a number of studies have demonstrated that PET outperforms CT in diagnosing nodal involvement in lung cancer $(3,5,6)$, PET could inevitably yield false-positive results (7). Recently, the combination of PET and CT (PET/CT) has significantly improved the accuracy and sensitivity of the lung cancer diagnosis $(3,8)$ and has become a routine aspect of the contemporary radiotherapy treatment planning process (9). However, the target delineation criteria have not yet been fully optimized (10) due to low image resolution and the difficulty in fusing the PET and CT images.

Diffusion-weighted magnetic resonance imaging (DW-MRI) can detect the restricted diffusion of water molecules through exploiting the random motion of water protons in biological tissue (11). Compared with normal tissues, malignant tumors exhibit a significantly decreased diffusion of water molecules. The difference in the diffusion of water molecules among tissues enables DW-MRI to detect malignant tumors and differentiate them from benign masses (12). Previous studies have shown that DW-MRI is comparable with PET/CT in detecting malignant lesions (13-15). Numerous studies reported that DW-MRI provided more accurate delineation for a number of cancer types, including prostate, head and neck, and cranial tumors (16-18). Compared with PET/CT, DW-MRI has demonstrated the same level of sensitivity or higher in detecting the nodal and primary malignancies in 
lung cancer (12). Nevertheless, the implication of DW-MRI in lung cancer, particularly lung cancer with pulmonary atelectasis, has not yet been intensively investigated. In the present study, the potential implication of DW-MRI in assessing central lung cancer with pulmonary atelectasis was evaluated and the successful implementation of DW-MRI in precision radiotherapy treatment planning was demonstrated.

\section{Patients and methods}

Patient selection. The patients recruited in the present study were histologically diagnosed with central lung cancer accompanied with pulmonary atelectasis. In general, all patients exhibited a good health condition with a Karnofsky performance status of $\geq 70$ (19) and with no contraindications to MRI examination. The images from CT, PET/CT and MRI scans for each individual patient were collected within 1 week. The results from the PET/CT scan were used as the standard for diagnosis. All patients provided written informed consent prior to enrolling in the study. The study was approved by the Institutional Review Board and the Ethics Committee of Shandong Cancer Hospital and Institute (Jinan, China).

Population. A total of 27 patients with central lung cancer without any antitumor therapy scheduled to receive precision radiotherapy were enrolled in the present study between October 2014 and June 2015. The cohort included 23 males and 4 females. The patient ages ranged from 37 to 79 years, with a median of 61 years. All patients were histologically confirmed with central lung cancer via biopsy. The cohort included 12 cases of squamous cell carcinoma, 6 cases of adenocarcinoma, 6 cases of small cell carcinoma, 2 cases of atypical carcinoid and 1 case of adenoid cystic carcinoma. The tumors were located in the upper left lung in 8 cases, the lower left lung in 4 cases, the upper right lung in 5 cases, the middle right lung in 4 cases and the lower right lung in 6 cases.

CT scan and image acquisition. Prior to radiotherapy, all patients underwent CT simulation using immobilization by an evacuated vacuum-bag in the supine position and a CT scanner (Brilliance CT Big Bore; Philips Healthcare, DA Best, The Netherlands), with a 3-mm slice thickness from the circular cartilage to the upper pole of the kidney. Following the scan, each patient was injected with $90 \mathrm{ml}$ ioversol $(320 \mathrm{mg} / \mathrm{ml})$ for enhancement scanning.

PET/CT scans and image acquisition. The PET/CT scan was performed with the GE Discovery LS PET/CT scanning system (GE Healthcare Life Sciences, Shanghai, China). All patients were requested to fast at least $6 \mathrm{~h}$ and it was necessary that their blood glucose should be within in the normal range prior to the $\mathrm{PET} / \mathrm{CT}$ examination. Between 40 and $60 \mathrm{~min}$ after the intravenous injection of fluorodeoxyglucose $\left({ }^{18} \mathrm{~F}-\mathrm{FDG} ; 5.55-7.40 \mathrm{MBq} / \mathrm{kg}\right.$ ), the CT scan was performed and emission images were acquired. The patient was immobilized by evacuated vacuum-bag in the supine position using the fixed-field parameters based on the CT. The PET images were reconstructed using the Ordered-Subset Expectation Maximization (OSEM) with the built-in software on the scanning machine, and the PET images were attenuation corrected with CT.

DW-MRI scan and image acquisition. The DW-MRI scan was performed by the Achieva 3.0T MR PHILIPS scanner (Philips Healthcare). Patients were kept in the same supine position as for the CT scan. The scanning parameters were as follows: i) Cross sectional T1-weighted image (T1WI): Repetition time/echo time TR/TE $10 \mathrm{sec} / 2 \mathrm{msec}$; slice thickness/interslice gap, 3/0 mm; field of view (FOV), $375 \mathrm{~mm}$; matrix, $352 \times 160$; ii) cross-sectional T2WI:TR/TE $1.5 \mathrm{sec} / 80 \mathrm{msec}$; slice thickness/interslice gap, 3/0 mm; FOV, $375 \mathrm{~mm}$; matrix, $352 \times 160$; iii) coronal T2WI: TR/TE, $1.8 \mathrm{sec} / 80 \mathrm{msec}$; slice thickness/interslice gap, 3/0 mm; FOV, $375 \mathrm{~mm}$; matrix, 352x160; and iv) DWI: TR/TE, $2.6 \mathrm{sec} / 52 \mathrm{msec}$; slice thickness/interslice gap, 3/0 mm; FOV, 375 mm; matrix, 352x160; diffusion-weighted sequence $\left(b=600 \mathrm{sec} / \mathrm{mm}^{2}\right)$ was added in the axial plane.

Image fusion. To delineate GTVs, all CT, PET and DW-MRI images were transferred to a treatment planning system (TPS, Eclipse V11.5; Varian Medical Systems, Palo Alto, CA, USA). The PET and DW-MRI images from each patient were fused with the corresponding CT images. The accuracy of registration was visually inspected using the software provided by the TPS.

Using the large aperture static image as the reference, the rigid registration of the gray scale image method, together with the correction based on the skeletal signs, was used to locate the PET and DW-MRI images in the coordinate system.

GTV delineation on CT, PET/CT and DW-MRI images. In total, 10 radiotherapists independently reviewed the CT, PET and DW-MRI images, and delineated the contours of the tumor following the standard procedure. The lung cancer usually shows a heterogeneous and lobulated mass with rough edges in CT image and the tumor edges were used as the reference on $\mathrm{GTV}_{\mathrm{CT}}$ delineation.

The ${ }^{18} \mathrm{~F}-\mathrm{FDG}$ concentration and characteristics of the lung lesions were used to determine the tumor tissue and the lung tissue on the PET/CT images. Using Eclipse V11.5, the contour of the primary tumor target zone in $\mathrm{GTV}_{\mathrm{PET}}$ was first automatically outlined if the standardized uptake value was $\geq 2.5$, while the non-tumor regions could manually be removed by referencing the CT image. On DW-MRI images, the solid region of the tumor appeared to have high signal intensity. By contrast, the pulmonary atelectasis and the obstructive inflammation had relatively low signal intensity. Only the area with a high signal was used in contouring $\mathrm{GTV}_{\mathrm{MRI}}$. The volumes of the target area were automatically provided using Eclipse V11.5 software.

Distance between centroids of GTVs. The three directional coordinates of the $\mathrm{GTV}_{\mathrm{CT}}, \mathrm{GTV}_{\mathrm{PET}}$ and $\mathrm{GTV}_{\mathrm{MRI}}$ centroids were determined using the Eclipse V11.5 software and were denoted as $(\mathrm{x}, \mathrm{y}, \mathrm{z})$. The relative coordinates between two GTV centroids were denoted as $(\Delta \mathrm{x}, \Delta \mathrm{y}, \Delta \mathrm{z})$, with $\Delta \mathrm{x}$ the distance in the left/right (LR) direction, $\Delta y$ the distance in the superior/inferior direction direction and $\Delta \mathrm{z}$ the distance in the anterior/posterior direction. The formula $\mathrm{V}=\sqrt{ }\left(\Delta \mathrm{x}^{2}+\Delta \mathrm{y}^{2}+\Delta \mathrm{z}^{2}\right)$ was used to 
Table I. GTV measurements using CT, PET/CT and diffusion-weighted MRI ( $\mathrm{n}=27)$.

\begin{tabular}{lcc}
\hline GTV measurements, $\mathrm{cm}^{3}$ & Mean (standard error of the mean) & P-value $^{\mathrm{a}}$ \\
\hline $\mathrm{GTV}_{\mathrm{CT}}$ & $109.45(14.90)$ & $\mathrm{N} / \mathrm{A}$ \\
$\mathrm{GTV}_{\mathrm{PET}}$ & $85.23(13.10)$ & $\mathrm{N} / \mathrm{A}$ \\
$\mathrm{GTV}_{\mathrm{MRI}}$ & $83.10(14.26)$ & $\mathrm{N} / \mathrm{A}$ \\
$\mathrm{GTV}_{\mathrm{CT}}-\mathrm{GTV}_{\mathrm{MRI}}$ & $26.34(6.39)$ & $<0.001$ \\
$\mathrm{GTV}_{\mathrm{CT}}-\mathrm{GTV}_{\mathrm{PET}}$ & $24.22(5.84)$ & 0.003 \\
$\mathrm{GTV}_{\mathrm{PET}}-\mathrm{GTV}_{\mathrm{MRI}}$ & $2.12(2.46)$ & 0.395
\end{tabular}

${ }^{a}$ GTV measurements were compared by Student's t-test. CT, computed tomography; PET, positron emission tomography; MRI, diffusion-weighted magnetic resonance imaging; GTV, gross target volume; N/A, not applicable.
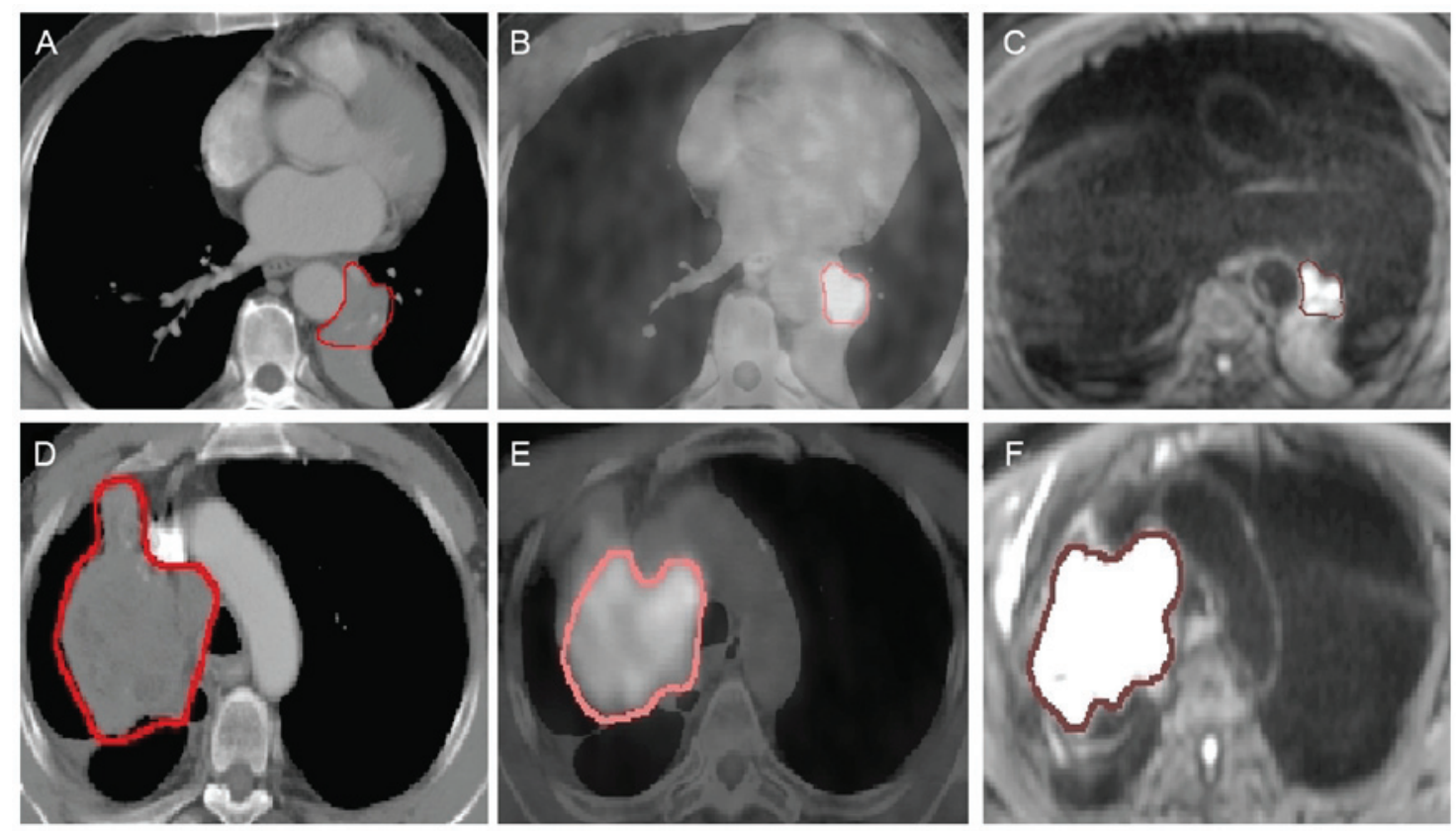

Figure 1. Delineation of CT, PET/CT and DW-MRI images. (A-C) Images of central lung cancer (squamous cell carcinoma) in a 74-year-old male patient. (D-F) Images of central lung cancer (small cell tumor) in a 61-year-old male patient. (A) The lower left lung tumor was accompanied with atelectasis, which results in blurry tumor edges. The $\mathrm{GTV}_{\mathrm{CT}}$ is outlined in red. (B) PET/CT image of the concentrated radioactive tracers in the left lower lung. The GTV $\mathrm{PET}_{\text {is }}$ outlined in pink. (C) DW-MRI of the lung tumor with clear edges. The signal from the atelectasis is relatively low, which can be easily differentiated from the real tumor tissues. The $\mathrm{GTV}_{\mathrm{MRI}}$ is outlined in brown. (D) CT image of the lung tumor. The GTV $\mathrm{CT}_{\mathrm{C}}$ is outlined in red. (E) PE/CT image of the concentrated radioactive tracers in the lung tumor. The $\mathrm{GTV}_{\mathrm{PET}}$ is outlined in pink. (F) DW-MRI of the lung tumor. The GTV $\mathrm{MRI}_{\mathrm{S}}$ is outlined in brown. CT, computed tomography; PET, positron emission tomography; DW-MRI, diffusion-weighted magnetic resonance imaging; GTV, gross target volume.

calculate the distance between centroids of GTVs (i.e., $\mathrm{GTV}_{\mathrm{CT}}$ vs. GTV $\mathrm{PET}_{\mathrm{P}}$; GTV $\mathrm{PET}_{\mathrm{PS}}$ VS. GTV $\mathrm{MRI}$; and $\mathrm{GTV}_{\mathrm{PET}}$ Vs. GTV

Statistical analysis. Statistical analysis was performed using the SAS 9.3 software (SAS Institute Inc., Cary, NC, USA). All the parameters were descriptively summarized, including the mean and standard deviation. The differences between the GTVs and the distance to the centroids of the GTVs are presented as the mean and standard error of the mean, and were assessed using Student's t-test. The difference was considered as statistically significant if $\mathrm{P}<0.05$. Pearson's correlation coefficient values were summarized for the GTVs measured by three different approaches. The comparison between the group means was performed using one-way analysis of variance. The variation in the target volume of the tumors measured by different radiotherapists was assessed by the variation coefficient $(\mathrm{CV})$ as follows: $\mathrm{CV}=$ standard deviation/mean x 100 .

\section{Results}

Collection of CT, PET/CT and DW-MRI images. To implement DW-MRI as a preferred lung cancer radiotherapy procedure that is reproducible, non-invasive and cost-effective, the present study evaluated and compared CT, PET/CT and DW-MRI images of lung cancer. A total of 27 patients were diagnosed with lung cancer and images were collected according to the aforementioned methods. Using image fusions, GTVs for CT, PET/CT and DW-MRI images were delineated. Images from 2 individual patients are presented as examples in Fig. 1.

The delineated tumors among the 27 subjects varied in shape and size. Noticeably and as expected, atelectasis 
compromised the precise delineation of the tumors in the majority of cases, as demonstrated by the blurry boundary in Fig. 1A and D. It is also worth noting that compared with the GTVs of the CT and PET/CT images, the delineated GTV of the DW-MRI images was often smaller in size with clear edges (Fig. 1C and F).

Pairwise comparison between $G T V_{C T}$, GTV $V_{P E T}$ and $G T V_{M R I}$. A total of 27 GTV measurements were obtained for all patients based on CT, PET/CT and DW-MRI images, respectively. $\mathrm{GTV}_{\text {Ст }}$ values ranged from 13.48 to $258.75 \mathrm{~cm}^{3}$, with a mean of $109.45 \mathrm{~cm}^{3}$, whereas $\mathrm{GTV}_{\mathrm{PET}}$ values ranged from 2.48 to $219.97 \mathrm{~cm}^{3}$, with a mean of $85.23 \mathrm{~cm}^{3}$, and $\mathrm{GTV}_{\mathrm{MRI}}$ values ranged from 3.88 to $246.95 \mathrm{~cm}^{3}$, with a mean of $83.10 \mathrm{~cm}^{3}$ (Table I). Among the 27 subjects analyzed, 23 subjects presented with a larger mean $\mathrm{GTV}_{\mathrm{CT}}$ than mean GTV $_{\text {MRI }}$ value, 22 subjects with a larger mean GTV $_{\text {CT }}$ than $\mathrm{GTV}_{\mathrm{PET}}$ value, and 21 subjects with a larger mean $\mathrm{GTV}_{\mathrm{PET}}$ than mean $\mathrm{GTV}_{\mathrm{MRI}}$ value (data not shown).

The mean $\mathrm{GTV}_{\text {Ст }}$ was larger than the mean GTV $_{\text {MRI }}$ and $\mathrm{GTV}_{\mathrm{PET}}$ by a value of $26.34 \mathrm{~cm}^{3}$ and $24.22 \mathrm{~cm}^{3}$, respectively (Table I). Student's t-test showed that the differences in the mean GTV between CT and MRI, and between CT and PET, were statistically significant (Table I), suggesting that CT contouring is statistically different from PET and MRI contouring. By contrast, the difference in the mean GTV between PET and MRI was negligible and not significantly different (Table I).

Correlations of $G T V_{C T}, G T V_{P E T}$ and $G T V_{M R I}$. The correlations among GTVs were also examined by measuring the Pearson's correlation coefficient in a pairwise manner. Pearson's correlation values of the three comparisons were all greater than 0.8 (Fig. 2), suggesting that all three contour methods are coherently related. However, Pearson's correlation between $\mathrm{GTV}_{\mathrm{PET}}$ and $\mathrm{GTV}_{\mathrm{MRI}}$ (Fig. 2A, r=0.9546) was significantly higher than those between $\mathrm{GTV}_{\mathrm{CT}}$ and $\mathrm{GTV}_{\mathrm{PET}}$ (Fig. 2B, r=0.8056), and between $\mathrm{GTV}_{\mathrm{CT}}$ and $\mathrm{GTV}_{\mathrm{MRI}}$ (Fig. 2C, $\mathrm{r}=0.8443)$. This demonstrates a direct dependency between PET and MRI contouring, and a divergence of CT from the other contouring methods.

Pairwise comparison between $C V$ of $G T V_{C T}, G T V_{P E T}$ and $G T V_{M R \text { I }}$. To evaluate the robustness and the reproducibility of the DW-MRI procedure, cancer images of the 27 subjects were randomly collected by 10 individual medical doctors and the $\mathrm{CV}$ was calculated for CT, PET/CT and DW-MRI procedures, respectively. CV is a standardized measure of dispersion in a distribution. The $\mathrm{CV}$ among doctors for the $\mathrm{GTV}_{\mathrm{MRI}}$ procedure was $26.60 \%$, which is markedly smaller than the CVs for the $\mathrm{GTV}_{\mathrm{PET}}(32.00 \%)$ and $\mathrm{GTV}_{\mathrm{CT}}(33.76 \%)$ procedures. These data suggested that DW-MRI is the most robust and reproducible procedure in lung cancer radiotherapy.

Distance between centroids of GTVs. The mean distance between the centroids of $\mathrm{GTV}_{\mathrm{CT}}$ and $\mathrm{GTV}_{\mathrm{PET}}$ was $0.87 \mathrm{~cm}$ $(\mathrm{SD}, 0.54 \mathrm{~cm})$. The mean distance between the centroids of $\mathrm{GTV}_{\mathrm{MRI}}$ and $\mathrm{GTV}_{\mathrm{PET}}$ was $0.72 \mathrm{~cm}(\mathrm{SD}, 0.34 \mathrm{~cm})$. No statistically significant differences in the distances were observed.
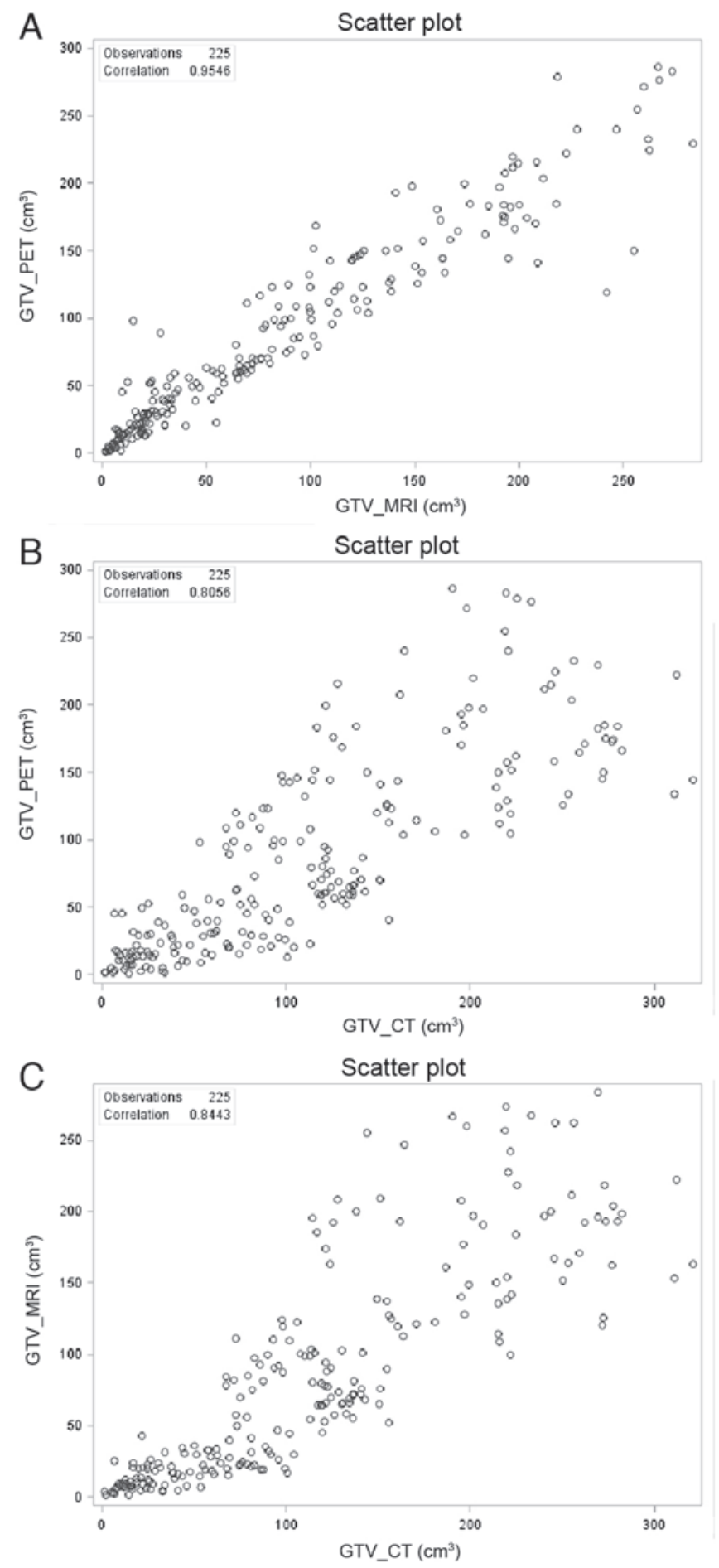

Figure 2. Scatter plots of GTV measurements using CT, PET/CT and DW-MRI procedures. (A) Pairwise comparison between GTV $_{\text {MRI }}$ and $\mathrm{GTV}_{\mathrm{PET}}$. (B) Pairwise comparison between $\mathrm{GTV}_{\mathrm{CT}}$ and $\mathrm{GTV}_{\mathrm{PET}}$. (C) Pairwise comparison between $\mathrm{GTV}_{\mathrm{CT}}$ and $\mathrm{GTV}_{\mathrm{MRI}}$. CT, computed tomography; PET, positron emission tomography; DW-MRI, diffusion-weighted magnetic resonance imaging; GTV, gross target volume.

\section{Discussion}

It is not uncommon that central lung cancer occurs with obstructive pulmonary atelectasis (20). Conceivably, accompanying atelectasis often obscures the accuracy of a central lung cancer diagnosis $(21,22)$. The present study observed the confounding effect of atelectasis on delineating the lung 
cancer (Fig. 1). Distinguishing central lung cancer from obstructive pulmonary atelectasis is critical in clinical staging and target delineation. An incorrect delineation of GTV could lead to decreased survival rates and increased side effects from radiotherapy (2).

A hallmark feature of an intensified CT scan is the superior spatial resolution of lung cancer. However, it is fairly challenging to distinguish lung cancer from pulmonary atelectasis due to inflammation and effusion $(23,24)$. Relatively low soft-tissue contrast prevents CT from providing precise information on the GTV extension in the vast majority of tissues. It has been widely recognized that this limitation of CT has led to inter- and intra-observer variations in GTV delineation in lung cancer $(25,26)$.

$\mathrm{PET} / \mathrm{CT}$ is an extremely important innovation in lung cancer imaging, which is capable of explicitly differentiating between the normal tissues and cancer tissues. PET/CT has been proven to significantly enhance the accuracy of conventional imaging in estimating the full spectrum of a number of tumors, including lung cancer (27). Numerous studies have reported that $\mathrm{PET} / \mathrm{CT}$ has an influential contribution on the radiotherapy of lung cancer patients $(24,28)$, as it can easily distinguish the central lung cancer from atelectasis.

MRI has been rapidly and widely deployed in radiotherapy planning due to its exquisite high contrast, high resolution soft tissue visualization and functional imaging modalities, which outperform PET/CT in terms of tumor visualization capability (26). It has been suggested that DWI combined with MRI can provide important information in differentiating lung cancer and atelectasis (20).

In the present study, the mean GTV measurements based on DW-MRI were significantly smaller than the mean GTV based on $\mathrm{CT}$, and these were indistinguishable from the mean GTV based on PET/CT (Table I), which is consistent with previous studies (17,29-32). DW-MRI is an excellent technique in differentiating the central lung cancer from obstructive pulmonary atelectasis and can provide accurate information for target delineation. In addition, it was shown that the distance between the centroids of CT and PET images was similar to that between the centroids of MRI and PET images, suggesting that the difference in GTVs using different procedures is largely attributable to the shape of the target delineation, but not the location of the target centers. Notably, delineation based on DW-MRI achieves similar clinical results with PET/CT, while it can avoid the radiation for patients during the PET/CT scan, as well as lowering the treatment cost.

The large variation among radiotherapists, and even for the same doctor over a period of time, is not uncommon in the target delineation $(33,34)$. The methodology defects and poor differentiation between normal tissues and tumors account for the inter-observer variations (26). In the present study, the variation based on DW-MRI was noticeably lower than that based on PET/CT and CT. Overall, DW-MRI is much more robust and reproducible compared with $\mathrm{CT}$ and PET/CT procedures. Due to relatively poor soft-tissue contrast of the CT image, it can only provide limited information for target delineation. GTV based on CT varied enormously between radiotherapists, although it was prophetically suggested that the variation could be decreased via rigid training. By contrast, the variations in estimating GTV using DW-MRI and PET/CT are much smaller and more stable among radiotherapists than that in CT. In other words, compared with PET/CT, DW-MRI has the highest target delineation precision and lowest variation to the same extent, thus significantly decreasing the toxicity of unintentional dosing and impairment of adjacent tissues during the radiotherapy. DW-MRI is highly recommended as it radiation-free and cost-effective, which is of particular benefit in developing countries.

\section{Acknowledgements}

This study was supported by the National Natural Science Foundation of China (grant nos. 81272699, 81301936 and 81472811), the Shandong Science and Technology Development Project (grant no. 2014GGC03038) and the International Cooperation Project of Science and Technology Department (grant no. 2012DFA31560).

\section{References}

1. Parkin DM, Bray F, Ferlay J and Pisani P: Global cancer statistics, 2002. CA Cancer J Clin 55: 74-108, 2005.

2. Senan S and De Ruysscher D: Critical review of PET-CT for radiotherapy planning in lung cancer. Crit Rev Oncol Hematol 56: 345-351, 2005.

3. Dwamena BA, Sonnad SS, Angobaldo JO and Wahl RL: Metastases from non-small cell lung cancer: Mediastinal staging in the 1990s-meta-analytic comparison of PET and CT. Radiology 213: 530-536, 1999.

4. Devic S: MRI simulation for radiotherapy treatment planning. Med Phys 39: 6701-6711, 2012.

5. Toloza EM, Harpole L and McCrory DC: Noninvasive staging of non-small cell lung cancer: A review of the current evidence. Chest 123 (Suppl 1): 137S-146S, 2003.

6. Gould MK, Kuschner WG, Rydzak CE, Maclean CC, Demas AN, Shigemitsu H, Chan JK and Owens DK: Test performance of positron emission tomography and computed tomography for mediastinal staging in patients with non-small-cell lung cancer: A meta-analysis. Ann Intern Med 139: 879-892, 2003.

7. Roberts PF, Follette DM, von Haag D, Park JA, Valk PE, Pounds TR and Hopkins DM: Factors associated with false-positive staging of lung cancer by positron emission tomography. Ann Thorac Surg 70: 1154-1160, 2000.

8. Silvestri GA, Gould MK, Margolis ML, Tanoue LT, McCrory D, Toloza E and Detterbeck F; American College of Chest Physicians: Noninvasive staging of non-small cell lung cancer: ACCP evidenced-based clinical practice guidelines (2nd edition). Chest 132 (Suppl 3): 178S-201S, 2007.

9. Al-Jahdali $\mathrm{H}$, Khan AN, Loutfi S and Al-Harbi AS: Guidelines for the role of FDG-PET/CT in lung cancer management. J Infect Public Health 5 (Suppl 1): S35-S40, 2012.

10. Aristei C, Falcinelli L, Palumbo B and Tarducci R: PET and PET-CT in radiation treatment planning for lung cancer. Expert Rev Anticancer Ther 10: 571-584, 2010.

11. Le Bihan D, Breton E, Lallemand D, Aubin ML, Vignaud J and Laval-Jeantet M: Separation of diffusion and perfusion in intravoxel incoherent motion MR imaging. Radiology 168: 497-505, 1988.

12. Usuda K, Zhao XT, Sagawa M, Matoba M, Kuginuki Y, Taniguchi M, Ueda Y and Sakuma T: Diffusion-weighted imaging is superior to positron emission tomography in the detection and nodal assessment of lung cancers. Ann Thorac Surg 91: 1689-1695, 2011.

13. Komori T, Narabayashi I, Matsumura K, Matsuki M, Akagi H, Ogura Y, Aga F and Adachi I: 2-[Fluorine-18]-fluoro-2-deoxy-D-gl ucose positron emission tomography/computed tomography versus whole-body diffusion-weighted MRI for detection of malignant lesions: Initial experience. Ann Nucl Med 21: 209-215, 2007.

14. Nomori H, Mori T, Ikeda K, Kawanaka K, Shiraishi S, Katahira K and Yamashita Y: Diffusion-weighted magnetic resonance imaging can be used in place of positron emission tomography for $\mathrm{N}$ staging of non-small cell lung cancer with fewer false-positive results. J Thorac Cardiovasc Surg 135: 816-822, 2008. 
15. Klingensmith WC III, Perlman D and Baum K: Intrapatient comparison of 2-deoxy-2-[F-18]fluoro-D-glucose with positron emissiontomography/computed tomography to Tc-99 m fanolesomab (NeutroSpec) for localization of infection. Mol Imaging Biol 9: 295-299, 2007.

16. Subesinghe M, Scarsbrook AF, Sourbron S, Wilson DJ, McDermott G, Speight R, Roberts N, Carey B, Forrester R, Gopal SV, et al: Alterations in anatomic and functional imaging parameters with repeated FDG PET-CT and MRI during radiotherapy for head and neck cancer: A pilot study. BMC Cancer 15: 137, 2015.

17. Sander L, Langkilde NC, Holmberg M and Carl J: MRI target delineation may reduce long-term toxicity after prostate radiotherapy. Acta Oncol 53: 809-814, 2014.

18. Navarria P, Reggiori G, Pessina F, Ascolese AM, Tomatis S Mancosu P, Lobefalo F, Clerici E, Lopci E, Bizzi A, et al: Investigation on the role of integrated PET/MRI for target volume definition and radiotherapy planning in patients with high grade glioma. Radiother Oncol 112: 425-429, 2014.

19. Karnofsky DA, Abelman WH, Craver LF and Burchenal JH: The use of nitrogen mustards in the palliative treatment of carcinoma. With particular reference to bronchogenic carcinoma. Cancer 1: 634-656, 1948

20. Qi LP, Zhang XP, Tang L, Li J, Sun YS and Zhu GY: Using diffusion-weighted MR imaging for tumor detection in the collapsed lung: A preliminary study. Eur Radiol 19: 333-341, 2009.

21. Yang RM, Li L, Wei XH, Guo YM, Huang YH, Lai LS, Chen AM, Liu GS, Xiong WF, Luo LP and Jiang XQ: Differentiation of central lung cancer from atelectasis: Comparison of diffusion-weighted MRI with PET/CT. PLoS One 8: e60279, 2013.

22. Onitsuka H, Tsukuda M, Araki A, Murakami J, Torii Y and Masuda K: Differentiation of central lung tumor from postobstructive lobar collapse by rapid sequence computed tomography. J Thorac Imaging 6: 28-31, 1991.

23. McAdams HP, Erasums JJ, Patz EF, Goodman PC and Coleman RE: Evaluation of patients with round atelectasis using 2-[18F]-fluoro-2-deoxy-D-glucose PET. J Comput Assist Tomogr 22: 601-604, 1998 .

24. Schmidt S, Nestle U, Walter K, Licht N, Ukena D, Schnabel K and Kirsch CM: Optimization of radiotherapy planning for non-small cell lung cancer (NSCLC) using 18FDG-PET. Nuklearmedizin 41: 217-220, 2002 (In German).

25. Senan S, van Sörnsen de Koste J, Samson M, Tankink H, Jansen P, Nowak PJ, Krol AD, Schmitz P and Lagerwaard FJ: Evaluation of a target contouring protocol for 3D conformal radiotherapy in non-small cell lung cancer. Radiother Oncol 53: 247-255, 1999.
26. Van de Steene J,Linthout N, de Mey J, Vinh-Hung V, Claassens C, Noppen M, Bel A and Storme G: Definition of gross tumor volume in lung cancer: Inter-observer variability. Radiother Oncol 62: 37-49, 2002.

27. Al-Jahdali H, Khan AN, Loutfi S and Al-Harbi AS: Guidelines for the role of FDG-PET/CT in lung cancer management. J Infect Public Health 5 (Suppl 1): S35-S40, 2012.

28. Ling CC, Humm J, Larson S, Amols H, Fuks Z, Leibel S and Koutcher JA: Towards multidimensional radiotherapy (MD-CRT): Biological imaging and biological conformality. Int J Radiat Oncol Biol Phys 47: 551-560, 2000.

29. Gao Z, Wilkins D, Eapen L, Morash C, Wassef Y and Gerig L: A study of prostate delineation referenced against a gold standard created from the visible human data. Radiother Oncol 85: 239-246, 2007.

30. McLaughlin PW, Evans C, Feng M and Narayana V: Radiographic and anatomic basis for prostate contouring errors and methods to improve prostate contouring accuracy. Int J Radiat Oncol Biol Phys 76: 369-378, 2010.

31. Seppälä T, Visapää H, Collan J, Kapanen M, Beule A, Kouri M, Tenhunen $M$ and Saarilahti K: Converting from CT- to MRI-only-based target definition in radiotherapy of localized prostate cancer: A comparison between two modalities. Strahlenther Onkol 191: 862-868, 2015.

32. Bradley J, Bae K, Choi N, Forster K, Siegel BA, Brunetti J, Purdy J, Faria S, Vu T, Thorstad W and Choy H: A phase II comparative study of gross tumor volume definition with or without PET/CT fusion in dosimetric planning for non-small-cell lung cancer (NSCLC): Primary analysis of radiation therapy oncology group (RTOG) 0515. Int J Radiat Oncol Biol Phys 82: 435-41.e1, 2012.

33. Tyng CJ, Chojniak R, Pinto PN, Borba MA, Bitencourt AG, Fogaroli RC, Castro DG and Novaes PE: Conformal radiotherapy for lung cancer: Interobservers' variability in the definition of gross tumor volume between radiologists and radiotherapists. Radiat Oncol 4: 28, 2009.

34. Rodríguez N, Sanz X, Trampal C, Foro P, Reig A, Lacruz M, Membrive I, Lozano J, Quera J and Algara M: 18F-FDG PET definition of gross tumor volume for radiotherapy of lung cancer: Is the tumor uptake value-based approach appropriate for lymph node delineation? Int J Radiat Oncol Biol Phys 78: 659-666, 2010. 